\title{
Evaluation of Spaceborne SiC Mirror Materials Using Samples Cut from the Periphery of a Mirror Body
}

\author{
Masaki Kotani, Yoshikazu Muta, Akinori Yoshimura, Shinji Ogihara, Tadashi Imai, Haruyoshi Katayama, Yukari Yui, Yoshio Tange, Keigo Enya, \\ Hidehiro Kaneda, and Takao Nakagawa
}

\author{
(Submitted October 9, 2013; in revised form November 11, 2013; published online December 21, 2013)
}

\begin{abstract}
The Japan Aerospace Exploration Agency has studied large-scale, lightweight mirrors constructed of SiCbased materials as a key technology for future earth observations and astronomical missions. One of the most important technical issues for large-scale ceramic components is their quality stability (viz., differences in material properties depending on the part and the processing), which might influence the structural and/or thermal reliability through unforeseen deformation and breakage. In this study, the authors used a simple, low-cost method for evaluating the properties of $\mathrm{SiC}$ mirror materials. Using mechanical testing, thermodilatometry, and microstructural analysis on samples cut from the periphery of a prototype 800mm-diameter mirror body, the overall quality of the mirror body material was determined.
\end{abstract}

Keywords coefficient of thermal expansion, material evaluation, microstructure, spaceborne $\mathrm{SiC}$ mirror, stiffness

\section{Introduction}

Future space missions for earth and astronomical observations demand improved observation capabilities for an onboard reflecting optical system. This upgrade requires larger primary mirrors with better specular accuracy as the key components of the system. Such mirrors must achieve the necessary mechanical and thermal reliability, while maintaining a lightweight structure. Hitherto, low thermal expansion glass $\left(1 \times 10^{-6} / \mathrm{K}\right)$ such as ZERODUR ${ }^{\circledR}$ and ULE $^{\circledR}$ has provided significant thermodimensional stability and hence has been used as the mirror body material in many spacecrafts. For example, the Hubble Space Telescope, launched in 1990, had a primary mirror of $2.4 \mathrm{~m}$ in diameter $\left(180 \mathrm{~kg} / \mathrm{m}^{2}\right)$, which enabled it to provide detailed information in research fields such as extrasolar planets, dark matter, and black holes. However, to actualize a large-diameter mirror $(\sim 2 \mathrm{~m})$ with a higher mirror accuracy $(\sim 100 \mathrm{~nm})$ and less weight $\left(\sim 50 \mathrm{~kg} / \mathrm{m}^{2}\right)$, new materials with higher specific stiffness (ratios of Young's modulus to specific weight) and adequate

Masaki Kotani and Akinori Yoshimura, Aerospace Research and Development Directorate, Japan Aerospace Exploration Agency, 6-131 Ohsawa, Mitaka-shi, Tokyo 181-0015, Japan; Yoshikazu Muta and Shinji Ogihara, Faculty of Science and Technology, Tokyo University of Science, 2641 Yamazaki, Noda-shi, Chiba 278-8510, Japan; Tadashi Imai, Haruyoshi, Katayama, Yukari Yui, and Yoshio Tange, Space Applications Mission Directorate, Japan Aerospace Exploration Agency, 2-1-1 Sengen, Tsukuba-shi, Ibaraki 305-8505, Japan; Keigo Enya and Takao Nakagawa, Institute of Space \& Astronautical Science, Japan Aerospace Exploration Agency, 3-1-1 Yoshinodai, Chuo-ku, Sagamihara-shi, Kanagawa 252-5210, Japan; and Hidehiro Kaneda, Graduate School of Science, Nagoya University, Furo-cho, Chikusa-ku, Nagoya-shi, Aichi 464-8602, Japan. Contact e-mail: kotani.masaki@jaxa.jp. thermal stability (ratio of thermal conductivity to coefficient of thermal expansion (CTE)) are needed. From the viewpoint of these performance indices, silicon carbide ( $\mathrm{SiC}$ ) materials are now attracting significant attention in the major countries sponsoring such space missions, and R\&D activities on this material are intensifying (Ref 1-4). The added benefits of using $\mathrm{SiC}$ include the maturity of the processing technique versus that for carbon-fiber-reinforced polymers (CFRPs) and the fact that it is safer to use in manufacturing than beryllium, which is currently used to fabricate large-area mirrors.

A SiC-based spaceborne primary mirror constructed of a porous $\mathrm{SiC}$ core coated with chemical-vapor-deposited $\mathrm{SiC}$ was first developed and launched in 2006 by Japan for the infrared astronomical satellite ASTRO-F (AKARI). The Herschel Space Observatory (HSO), launched in 2009 by the European Space Agency, was equipped with a segmented primary mirror with an aperture of $3.5 \mathrm{~m}$. The mirror was made of $\mathrm{SiC} 100^{\circledR}$, a $\mathrm{SiC}$ material developed by BOOSTEC ${ }^{\circledR}$ (France) and EADS Astrium that was produced using a sintering process. The Space Infrared Telescope for Cosmology and Astrophysics (SPICA), an infrared space telescope, is scheduled to be launched in 2022 by Japan in cooperation with other countries; its goal is to elucidate the birth and evolution of galaxies and to help researchers gain a comprehensive understanding of the formation process of planetary systems. SPICA will be equipped with a SiC-based primary mirror of $3.5 \mathrm{~m}$ in diameter whose mirror accuracy is higher than that of the HSO. The mirror will be made of a short, carbon-fiber-reinforced $\mathrm{C} / \mathrm{SiC}$ material called "HBCesic ${ }^{\circledR}$," developed by Engineered Ceramic Materials (ECM) $\mathrm{GmbH}$ (Germany) and Mitsubishi Electric Corporation (Japan), and produced by reaction bonding.

The Japan Aerospace Exploration Agency (JAXA) conducted cross-departmental research on the manufacturing and evaluation of large-scale, lightweight $\mathrm{SiC}$ mirrors as the key components for SPICA and other future earth observation and disaster-monitoring satellites. The research team examined the manufacturability of a large-scale, lightweight mirror body, a quality assurance process, and cryogenic mirror-surface testing technology by manufacturing and evaluating a prototype 
optical system with a primary mirror of $800 \mathrm{~mm}$ in diameter constructed entirely of HBCesic ${ }^{\circledR}$ (Ref 5-9). A schematic image of the optical system and a photograph of the 800 -mm-diameter primary mirror used in the experiment are shown in Fig. 1(a) and (b), respectively. This paper reports some of the results of the research conducted on the quality assurance process.

To meet the challenge of developing an unprecedentedly large mirror using a new material, it is necessary to clearly define the quality standards and establish an evaluation technology that can accurately verify that the developed mirror meets the required standard. To insure mechanical reliability, we need an evaluation method that can verify the absence of noticeable defects, and we also need to know the distribution of the related mechanical properties with high credibility. In terms of thermal reliability, we know that variations in thermal expansion will cause uneven thermal deformation, which will generate deviations in the desired mirror surface and thus affect the resulting images. These variations can be generated during the preparation or heating of the raw materials. Identifying these variations using nondestructive testing methods such as liquid penetrant methods, ultrasonic inspection, and so on is very difficult, more so than detecting defects. Thus, a method to
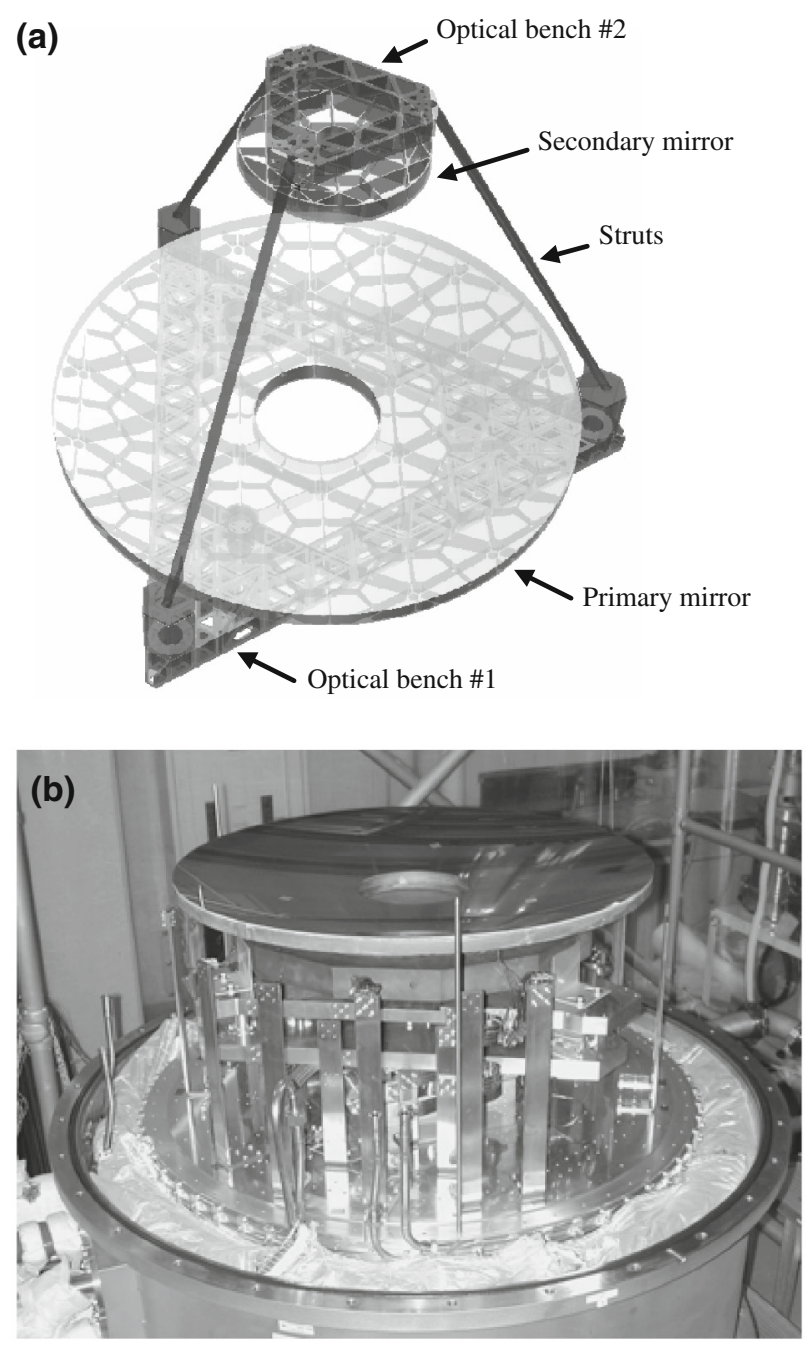

Fig. 1 (a) Schematic image of an optical system that includes the 800 -mm-diameter primary mirror and (b) photograph of the $\$ 800$ $\mathrm{mm}$ primary mirror in the experiment determine the distribution of thermal expansion properties without damaging the mirror body is necessary.

In this work, we tried to evaluate the material properties of spaceborne SiC mirrors by a method that does not rely on nondestructive testing. As a simple, low-cost measure, we used samples cut from the periphery of a block of a prototype 800-mmdiameter mirror body. We conducted mechanical tests, thermodilatometry, and microstructural analysis and examined the relationships among the various factors. The differences in the material microstructure and properties under various sampling conditions were identified and logically correlated. Consequently, the causes of the variations among the properties could be determined, and guidelines for improvement can be suggested. Finally, this study verified that this method of using cut samples is useful for understanding the characteristics of a large material block.

\section{SiC Mirror Material Evaluation}

The subject of this study was a block of material from an 800mm-diameter SiC mirror body (Fig. 1b). The mirror body was manufactured in a project of Mitsubishi Electric Corporation conducted jointly with Engineered Ceramic Materials $\mathrm{GmbH}$ (ECM) of Germany under a contract with JAXA. The material is based on HBCesic ${ }^{\circledR}$ (Ref 10), which was produced using the reaction sintering method and contained short carbon fibers for improved toughness (Ref 11-13). A mixture of a carbon precursor resin and short carbon fibers was fired to produce a porous carbon base material, which was then impregnated with molten silicon at a high temperature to induce reaction sintering to form a $\mathrm{SiC}$ phase. The positions and number indexes of the samples cut from this block of material are shown in Fig. 2 and Table 1.

To evaluate the mechanical properties, we measured the flexural strength and flexural elastic modulus using a threepoint flexural test with reference to the JIS R1601 and JIS R1602 standards, respectively. Rectangular test pieces that were $40 \mathrm{~mm}$ long, $4 \mathrm{~mm}$ wide, and $3 \mathrm{~mm}$ high were examined at room temperature by using a Model 5882 material testing system (Instron Corporation, USA). The load cell had a maximum capacity of $5 \mathrm{kN}$. The distance between the supporting points was $30 \mathrm{~mm}$, and the crosshead speed was $0.5 \mathrm{~mm} / \mathrm{min}$. To determine the deformation behavior of the test pieces, we measured their flexural strain by attaching a strain gage (KFG-5-120-C1-11L1M2R, Kyowa Electronic Instruments Co., Ltd., Japan) to the tension side under the load point of each test piece. We defined the maximum flexural stress supported by the test piece during testing as its flexural strength. We also computed the slope of the stress-strain curve at flexural stresses of 10-100 MPa by using the least-squares method and defined the slope as the flexural elastic modulus. Then, we evaluated the degree of variation in the flexural strength by Weibull analysis. The variations in the average values under various sampling conditions was computed as

$$
\begin{gathered}
(\mid \text { maximal value }|-| \text { minimum value } \mid) / \\
\mid \text { minimum value } \mid \times 100(\%)
\end{gathered}
$$

The thermal expansion properties were obtained by measuring the dimensional change of a test piece in one direction, while its temperature was increased at constant rate, as per the ISO 17562 standard, using a thermal dilatometer (DIL402C, NETZSCH-Geratebau GmbH, Germany). Rectangular test pieces that 


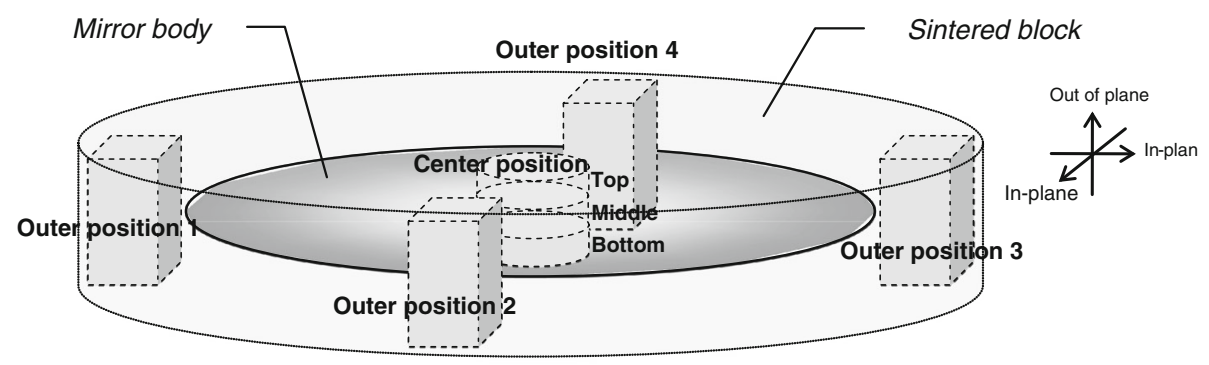

Fig. 2 Schematic illustration of the positions of samples cut out from the material block

Table 1 The positions and the number of samples cut out from the material block

\begin{tabular}{|c|c|c|c|}
\hline Usage & Location & Direction & Number \\
\hline \multirow[t]{10}{*}{ Mechanical test } & Center position & & \\
\hline & Upper part & In-plane & 10 \\
\hline & Middle part & In-plane & 50 \\
\hline & & Out-of-plane & 10 \\
\hline & Lower part & In-plane & 10 \\
\hline & Outer positions (middle part) & & \\
\hline & Position $1\left(0^{\circ}\right)$ & In-plane & 10 \\
\hline & Position $2\left(90^{\circ}\right)$ & In-plane & 10 \\
\hline & Position $3\left(180^{\circ}\right)$ & In-plane & 10 \\
\hline & Position $4\left(270^{\circ}\right)$ & In-plane & 10 \\
\hline \multirow[t]{14}{*}{ Thermodilatometry } & Center position & & \\
\hline & Upper part & In-plane & 5 \\
\hline & Middle part & In-plane & 5 \\
\hline & & Out-of-plane & 5 \\
\hline & Lower part & In-plane & 5 \\
\hline & Outer positions (middle part) & & \\
\hline & Position $1\left(0^{\circ}\right)$ & In-plane & 5 \\
\hline & & Out-of-plane & 5 \\
\hline & Position $2\left(90^{\circ}\right)$ & In-plane & 5 \\
\hline & & Out-of-plane & 5 \\
\hline & Position $3\left(180^{\circ}\right)$ & In-plane & 5 \\
\hline & & Out-of-plane & 5 \\
\hline & Position $4\left(270^{\circ}\right)$ & In-plane & 5 \\
\hline & & Out-of-plane & 5 \\
\hline \multirow[t]{9}{*}{ Microstructure examination } & Center position & & \\
\hline & Upper part & In-plane & 3 \\
\hline & Middle part & In-plane & 3 \\
\hline & Lower part & In-plane & 3 \\
\hline & Outer positions (middle part) & & \\
\hline & Position $1\left(0^{\circ}\right)$ & In-plane & 3 \\
\hline & Position $2\left(90^{\circ}\right)$ & In-plane & 3 \\
\hline & Position $3\left(180^{\circ}\right)$ & In-plane & 3 \\
\hline & Position $4\left(270^{\circ}\right)$ & In-plane & 3 \\
\hline
\end{tabular}

were $25 \mathrm{~mm}$ long, $6 \mathrm{~mm}$ wide, and $4 \mathrm{~mm}$ high were prepared while paying careful attention to the parallelism between the opposite faces in the longitudinal direction. A test piece was set in a holder made entirely from quartz glass and dried at $100{ }^{\circ} \mathrm{C}$ for more than $8 \mathrm{~h}$. Then, as a displacement detection rod was pressed into the test piece in the longitudinal direction at a fixed load of $15 \mathrm{cN}$, the temperature of the piece was increased at a constant rate of $3{ }^{\circ} \mathrm{C} / \mathrm{min}$ from 0 to $120{ }^{\circ} \mathrm{C}$ in a flow of high-purity helium at $100 \mathrm{~mL} / \mathrm{min}$. From the results, the apparatus automatically calculated the rate of dimensional change $\left(\Delta L / L_{0}\right)$ occurring in the test piece during the test. Here, $L_{0}$ is the length of the test piece at room temperature before the test and $\Delta L$ is the dimensional change in response to the change in temperature.

From the rate of dimensional change, we calculated the coefficient of linear expansion $\alpha$ and the average coefficient of linear expansion $\bar{\alpha}$, as expressed by formulas (1) and (2). Here, $\alpha$ was found by using the central difference method at 50,60, and $70{ }^{\circ} \mathrm{C}$ with $d T=20^{\circ} \mathrm{C}$, and $\bar{\alpha}$ corresponds to the slope of the straight line passing through two temperature points and was found in the range of $40-80{ }^{\circ} \mathrm{C}$.

$\alpha=\frac{1}{L_{0}} \cdot \frac{d L}{d T}$,

$\bar{\alpha}=\frac{\left(\frac{\Delta L}{L_{0}}\right)_{T_{1}}-\left(\frac{\Delta L}{L_{0}}\right)_{T_{2}}}{T_{1}-T_{2}}$.

Each test piece was measured twice in a row without disturbing the test piece, and the average of the two measurements 
was used for evaluation. The variations in the average values under various sampling conditions were computed as

( $\mid$ maximal value $|-|$ minimum value $\mid) /$

$\mid$ minimum value $\mid \times 100(\%)$.

Let us consider the accuracy of this measurement. Because the resolution of the detector and the longitudinal dimensions of the test piece were $0.125 \mathrm{~nm}$ and $25 \mathrm{~mm}$, respectively, the potential resolution for $\Delta L / L_{0}$ is $\pm 5.0 \times 10^{-9} /{ }^{\circ} \mathrm{C}(0.125 \mathrm{~nm} \div 25 \mathrm{~mm})$. Therefore, the measurement accuracies of $\alpha$ and $\bar{\alpha}$ can be estimated as $\pm 5.0 \times 10^{-10} /{ }^{\circ} \mathrm{C}\left(5.0 \times 10^{-9} \times 2 \div 20\right)$ and $\pm 2.5 \times 10^{-10} /{ }^{\circ} \mathrm{C}$ $\left(5.0 \times 10^{-9} \times 2 \div 40\right)$, respectively. As a preliminary verification of the device, a standard sample made of quartz glass was consecutively measured eight times without disturbing the test piece under the same conditions. As a result, we obtained $6.91 \times 10^{-9} /{ }^{\circ} \mathrm{C}$ as the standard deviation of $\bar{\alpha}$ in the range of 40-80 ${ }^{\circ} \mathrm{C}$. During the measurement of the test sample, we minimized the effects of opening/closing the sample room or detaching/attaching the test piece as much as possible by completely drying the sample, measuring one test piece twice, and averaging the measurement results for five test pieces under the same sampling conditions. These precautions secured an accuracy of $10^{-8} /{ }^{\circ} \mathrm{C}$.

From each position on the material block, a rectangular sample that was $5 \mathrm{~mm}$ long, $4 \mathrm{~mm}$ wide, and $2 \mathrm{~mm}$ high was cut as shown in Table 1. The surface parallel to the in-plane direction of the block was mirror polished, and the polished face was used as the sample for the following two microstructural evaluations.

The typical microstructure and composition distribution of the material were studied using a representative sample. Using an EPMA-1610 electron probe microanalyzer (Shimadzu Corporation, Japan), a backscattered electron image (BEI) and a secondary electron image (SEI) were acquired. Semiquantitative analysis of the composition of each phase and an elemental distribution analysis were also conducted.

Micrographs of the samples obtained under various sampling conditions were taken using a scanning electron microscope (SEM) and analyzed, and the volume fraction of each phase was determined. Using a field-emission scanning electron microscope (FE-SEM, S-4700, Hitachi High Technologies Corp., Japan), four micrographs were taken for each sample. To improve the discrimination accuracy in the image analysis for each phase, the images were preprocessed by adjusting the brightness, contrast, averaging phenomenon, blurring phenomenon, and so forth using Adobe ${ }^{\circledR}$ Photoshop ${ }^{\circledR}$ CS4 (Adobe Systems Inc., USA). These preprocessed images were analyzed to obtain the area ratios of the phases using the image analysis software Image-Pro ${ }^{\circledR}$ Plus version 6.2 (Media Cybernetics, Inc., USA). In order to discriminate the phases, the micrographs were first processed using the automatic identification function of the software and then manually modified. The obtained area ratio of each phase was raised to the power of three by two to obtain the volume fraction. After the average value of each sample was calculated, the average values were obtained for various sampling conditions.

\section{Experimental Results}

A typical stress-strain curve and an SEM image of the fracture surface obtained after the flexural testing of this material are shown in Fig. 3 and 4, respectively. In the stressstrain chart, the stress relative to the strain increased almost linearly, starting from the first application of the load until the sample fractured, which is typical behavior for ceramics. The fracture surface had more microbumps and dimples than are found in glass and high-density ceramics. These bumps and dimples are speculated to be mainly attributable to the material's microstructure, in which multiple phases formed by reaction sintering are deposited at random. These multiple phases might also have contributed somewhat to the heightened fracture resistance and fracture energy.

The flexural strengths and flexural elastic moduli obtained from the mechanical property tests under various sampling conditions are shown in Fig. 5. The error bars in the figure represent standard deviations. In addition, the degrees of variation in the average values obtained under various conditions are summarized in Table 2. The average values of the flexural strengths and flexural elastic moduli of all the samples were $330 \mathrm{MPa}$ and $316 \mathrm{GPa}$, respectively, which are common values for a $\mathrm{SiC}$ material. The flexural strength was higher in the outer positions than in the center position, whereas the flexural elastic modulus was slightly higher in the center position than in the outer positions. In the height direction, the flexural strength stayed almost the same, but the flexural elastic modulus was higher in the middle than at the top and bottom.

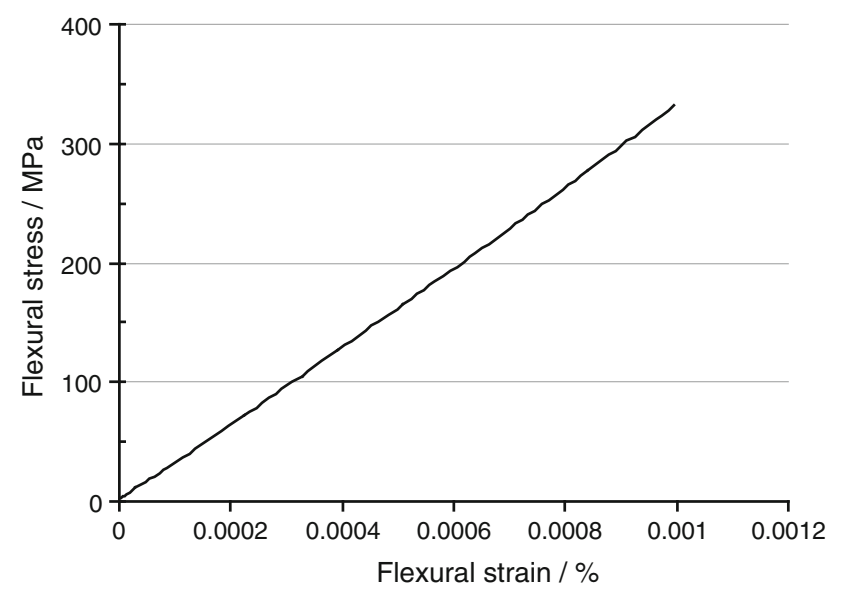

Fig. 3 Typical stress-strain chart of the sample obtained from the flexural test

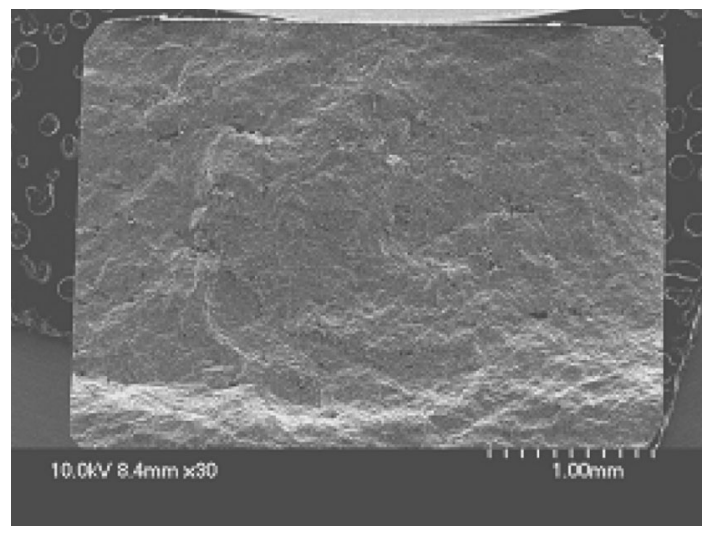

Fig. 4 Typical fracture surface of the sample observed after the flexural test 


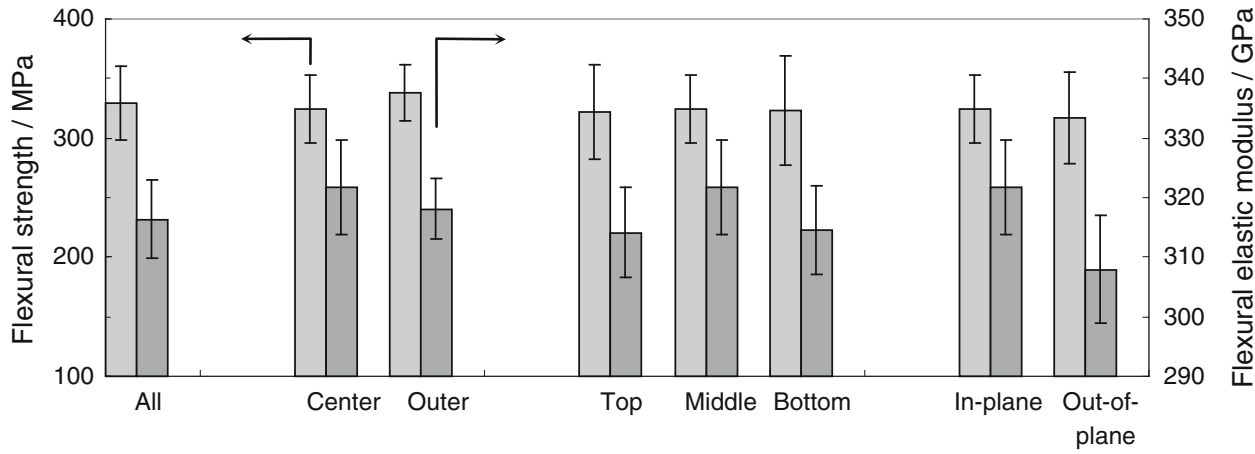

Fig. 5 Flexural strengths and flexural elastic moduli obtained from the flexural test under various sampling conditions

Table 2 Summary of the degrees of variation of flexural strength and flexural elastic modulus under various conditions

Sampling condition

Flexural strength, \%

Flexural elastic modulus, \%

Center versus outer

Upper versus middle versus lower

4.0

1.1

In-plane versus out-of-plane

$\begin{array}{ll}0.8 & 2.4 \\ 2.3 & 4.5\end{array}$

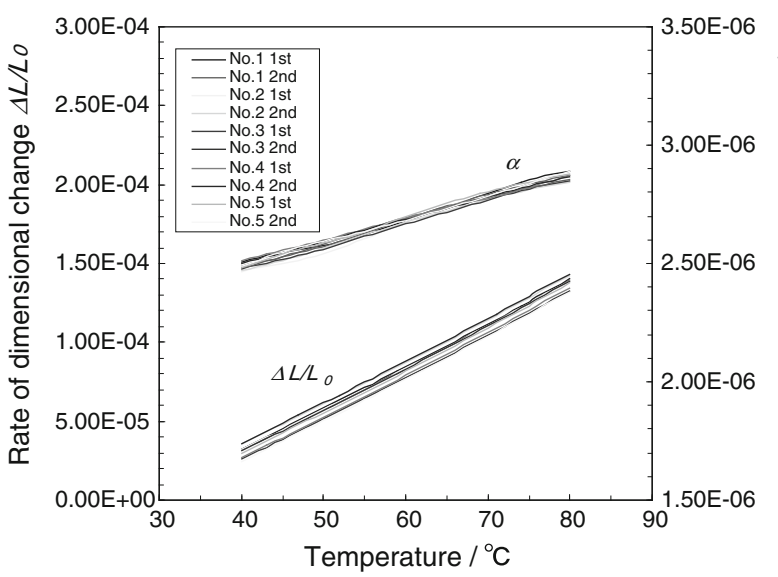

Fig. 6 Examples of the temperature trajectories of the rate of dimensional change $\Delta L / L_{0}$ and linear expansion coefficient $\alpha$ obtained from thermodilatometry

The flexural elastic modulus was higher in the in-plane direction than in the out-of-plane direction. The flexural strength differed by more than $4 \%$ between the center and the outer positions, and a similar difference appeared in the flexural elastic moduli between the in-plane and out-of-plane directions. The Weibull coefficient was 12.1 overall, which is a high value for a ceramic material. However, the values in the outer positions were higher than those in the center.

The temperature trends of $\Delta L / L_{0}$ and $\alpha$ obtained from the thermal expansion properties test are shown in Fig. 6. In the target temperature range, $\Delta L / L_{0}$ increased at a slightly accelerating rate as the temperature increased, and accordingly $\alpha$ increased almost linearly with temperature. The average values of all 60 samples (7 positions and 12 types) are shown in Fig. 7. For every increase of $10^{\circ} \mathrm{C}, \alpha$ increased almost linearly, from approximately $2.6 \times 10^{-6} /{ }^{\circ} \mathrm{C}$ to approximately $2.8 \times 10^{-6} /{ }^{\circ} \mathrm{C}$. The value of $\bar{\alpha}$ from 40 to $80{ }^{\circ} \mathrm{C}$ was $2.72 \times 10^{-6} /{ }^{\circ} \mathrm{C}$, which is approximately halfway between $2.6 \times 10^{-6}$ and $2.8 \times 10^{-6} /{ }^{\circ} \mathrm{C}$. The standard deviation was approximately $0.05 \times 10^{-6} /{ }^{\circ} \mathrm{C}$ at

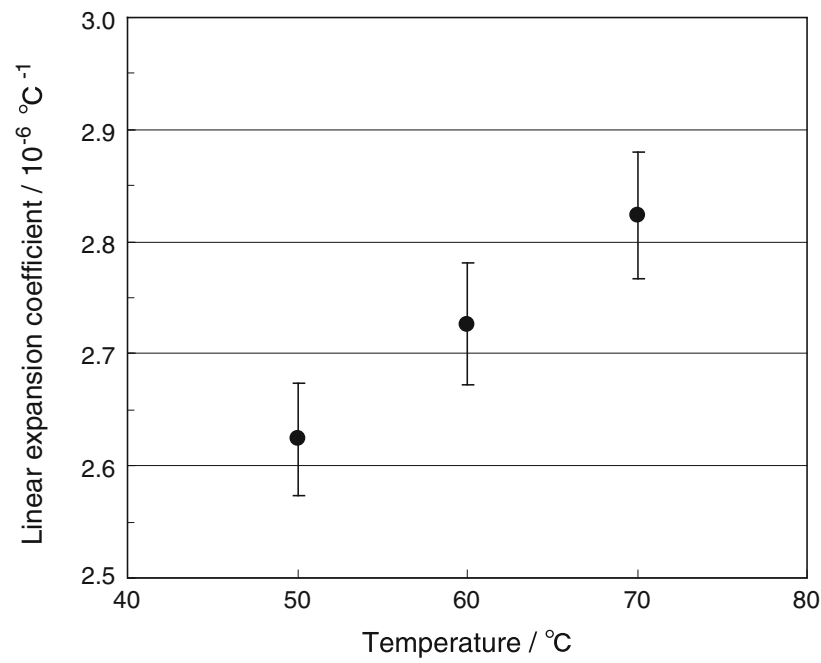

Fig. 7 Average $\alpha$ values of all 60 samples at 50,60 , and $70 \mathrm{~K}$

each temperature, and the relative standard deviation in $\bar{\alpha}$ was slightly less than $2 \%$.

The variation in the thermal expansion properties obtained under various sampling conditions was verified based on the individual $\bar{\alpha}$ values, and the results are shown in Fig. 8(a)-(c). The degrees of variation are summarized in Table 3. Relatively large variations were observed between the in-plane and out-ofplane directions at the center, in the height direction at the center, and between the center and the outer positions in the inplane direction.

Figure 9(a) shows the BEI of a representative cross section, which more clearly shows the difference in composition, and Fig. 9(b) shows a composite of the BEI and the distribution of $\mathrm{Si}$ and $\mathrm{C}$ from the same view. It can be observed that this material consists of three phases: a white phase, a phase that looks nearly black, and an intermediate gray phase. The white and black phases are never found side by side, and the gray phase is always present between the white and black phases. Long thin objects can also be observed in the black phase. 
From the element distribution chart on the right of Fig. 9(b), it can be observed that the black phase consists of $\mathrm{C}$, the white phase consists of $\mathrm{Si}$, and the gray phase consists of $\mathrm{Si}$ and $\mathrm{C}$. Further semiquantitative analysis revealed that the gray phase consisted of $\mathrm{SiC}$, in which the molar ratio of $\mathrm{Si}$ and $\mathrm{C}$ was approximately $1: 1$. Based on these findings, it is confirmed that the black, gray, and white phases are, respectively, green (not
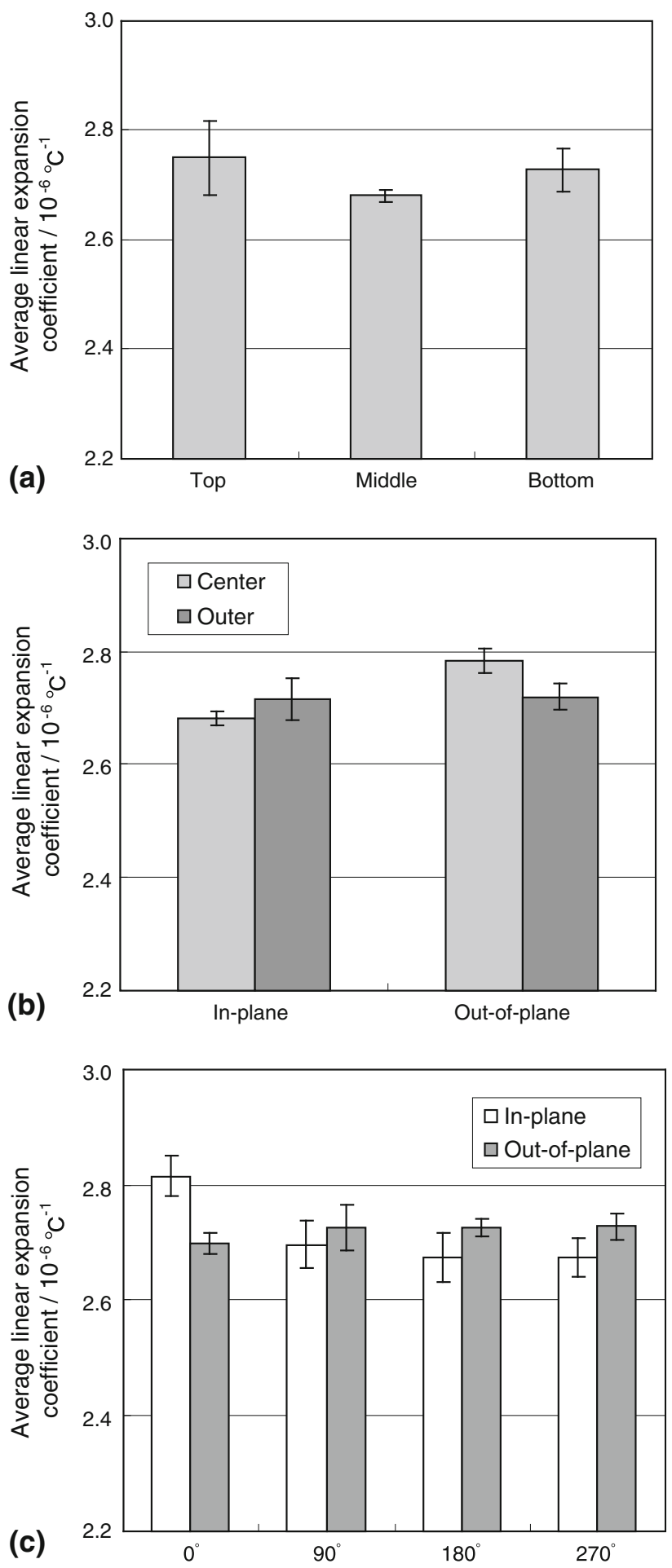

Fig. 8 Average linear expansion coefficients $\bar{\alpha}$ at $40-80{ }^{\circ} \mathrm{C}$ under various sampling conditions sintered) carbon, reaction-produced $\mathrm{SiC}$, and residual silicon. The long thin black objects are the short carbon fibers present in the green carbon body.

Similarly, the SEI of a representative cross section, which more clearly shows the surface aspects, is provided in Fig. 10. In this figure, micropores of several micrometers in diameter can be observed, although they are few in number. Figure 10(b) and (c) shows enlargements of regions A and B in Fig. 10(a), exhibiting the $\mathrm{C}$ and $\mathrm{Si}$ phases, respectively. The differences in the distributions of pores for different positions of the sample in a material block were not studied.

Figure 11 shows the volume fractions of different phases under various sampling conditions as obtained by the image analysis. At all positions, the volume fraction of the $\mathrm{SiC}$ phase was greater than $90 \%$, that of the Si phase was $6-8 \%$, and that of the $\mathrm{C}$ phase was less than $1 \%$. The combined volume fraction of $\mathrm{SiC}$ and $\mathrm{C}$ was relatively high in the center and middle parts (Fig. 11a and b, respectively).

\section{Discussion}

The $\mathrm{SiC}$ observed in this study is a reaction product of a carbon-based material and molten silicon. The volume fractions of the different phases indicate that most of the $\mathrm{C}$ in the base material encountered $\mathrm{Si}$ during sintering and became $\mathrm{SiC}$. It would appear from the distributions of the various phases, as shown in Fig. 9(b), that although molten $\mathrm{Si}$ thoroughly permeated the green carbon body during sintering to create $\mathrm{SiC}$, some short fibers with high crystallinity and/or parts located far from the surface of the carbon structure remained unreacted. We have estimated that the volume of $\mathrm{C}$ increased to about 1.9 times the original volume when $\mathrm{Si}$ (assuming a density of $2.3 \mathrm{~g} / \mathrm{cm}^{3}$ ) reacted with $\mathrm{C}$ (assuming a density of $1.8 \mathrm{~g} / \mathrm{cm}^{3}$ ) to become $\mathrm{SiC}$ (assuming a density of $3.2 \mathrm{~g} / \mathrm{cm}^{3}$ ). Then, from the volume fractions of $\mathrm{SiC}$ and $\mathrm{C}$, the filling fraction of $\mathrm{C}$ in the green body is estimated to be greater than $45 \%$. In addition, it is speculated that the pores in the $\mathrm{C}$ phase were formed from the pyrolytic gas from the precursor resin when the carbon-based material was produced and that the pores in the $\mathrm{Si}$ phase were formed by the inclusion of gas during Si impregnation.

Table 3 Summary of the degrees of variation of average linear expansion coefficients $\bar{\alpha}$ at $40-80{ }^{\circ} \mathrm{C}$ under various conditions

\begin{tabular}{lc}
\hline Sampling condition & Degree of variation, \% \\
\hline Upper versus middle versus lower & 2.5 \\
Center versus outer & 1.3 \\
$\quad$ In-plane & 2.3 \\
Out-of-plane & 0.6 \\
Average of in-plane and out-of-plane & \\
In-plane versus out-of-plane & 3.8 \\
Center & 0.2 \\
Outer & \\
Four positions at outer region & 5.3 \\
In-plane & 1.1 \\
Out-of-plane & 2.1 \\
Average of in-plane and out-of-plane & \\
\hline
\end{tabular}




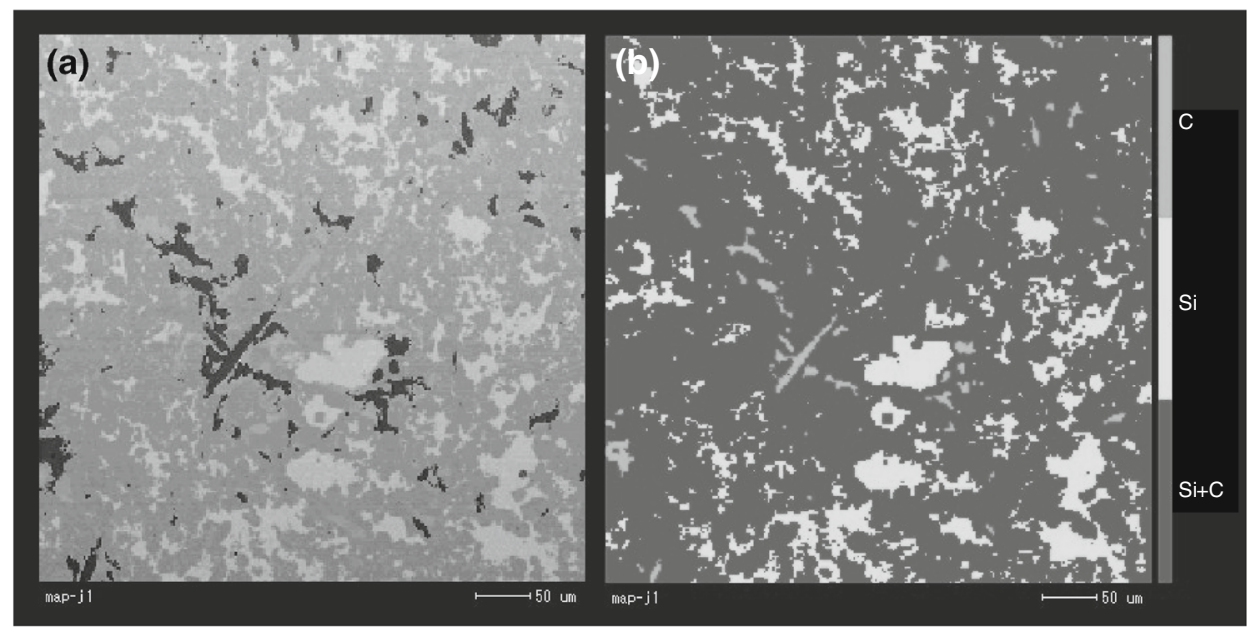

Fig. 9 (a) Backscattered electron image of a representative cross section of the sample and (b) composition distribution of Si and $\mathrm{C}$ of the same view

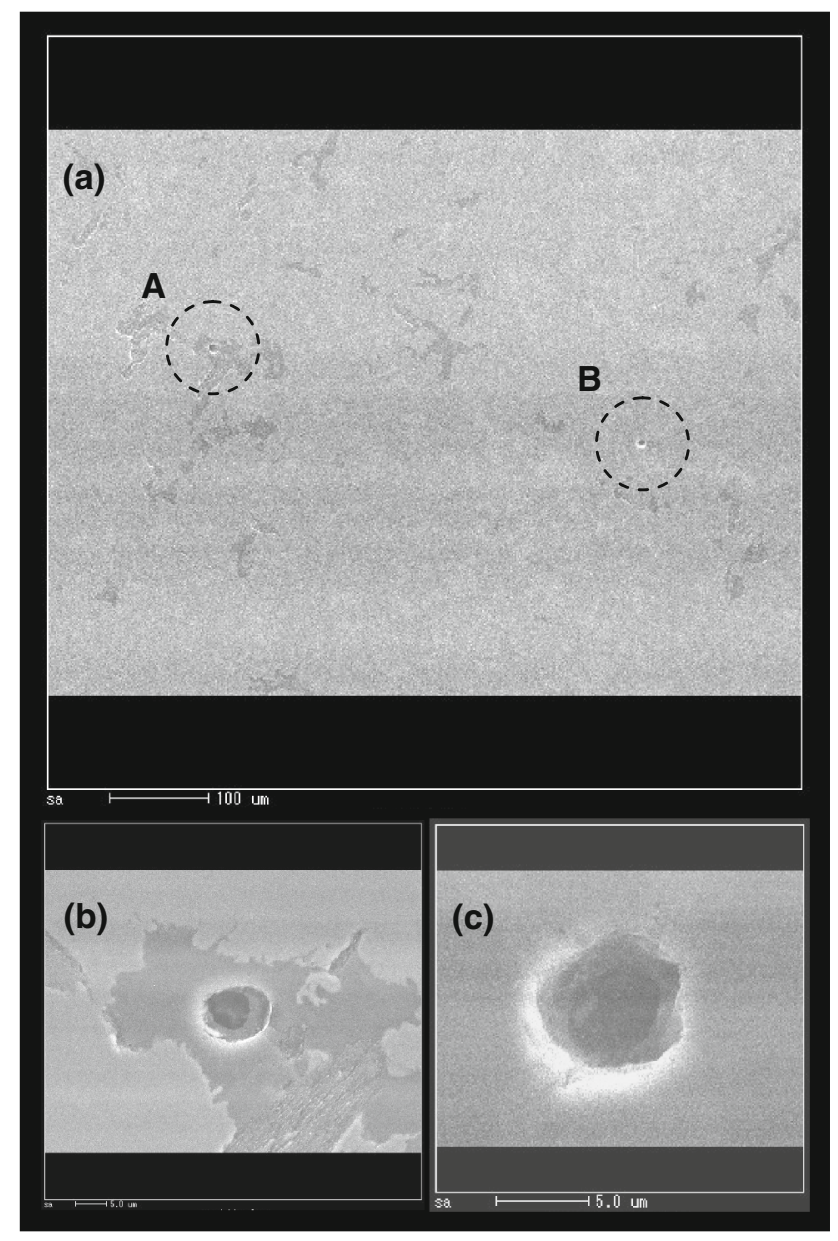

Fig. 10 Secondary electron images of a representative cross section of the sample

Because the elastic modulus was relatively high in the middle of the material block, it is presumed that microstructural conditions that elevated the elastic modulus were dominant in the central part. In addition, because both the strength and the
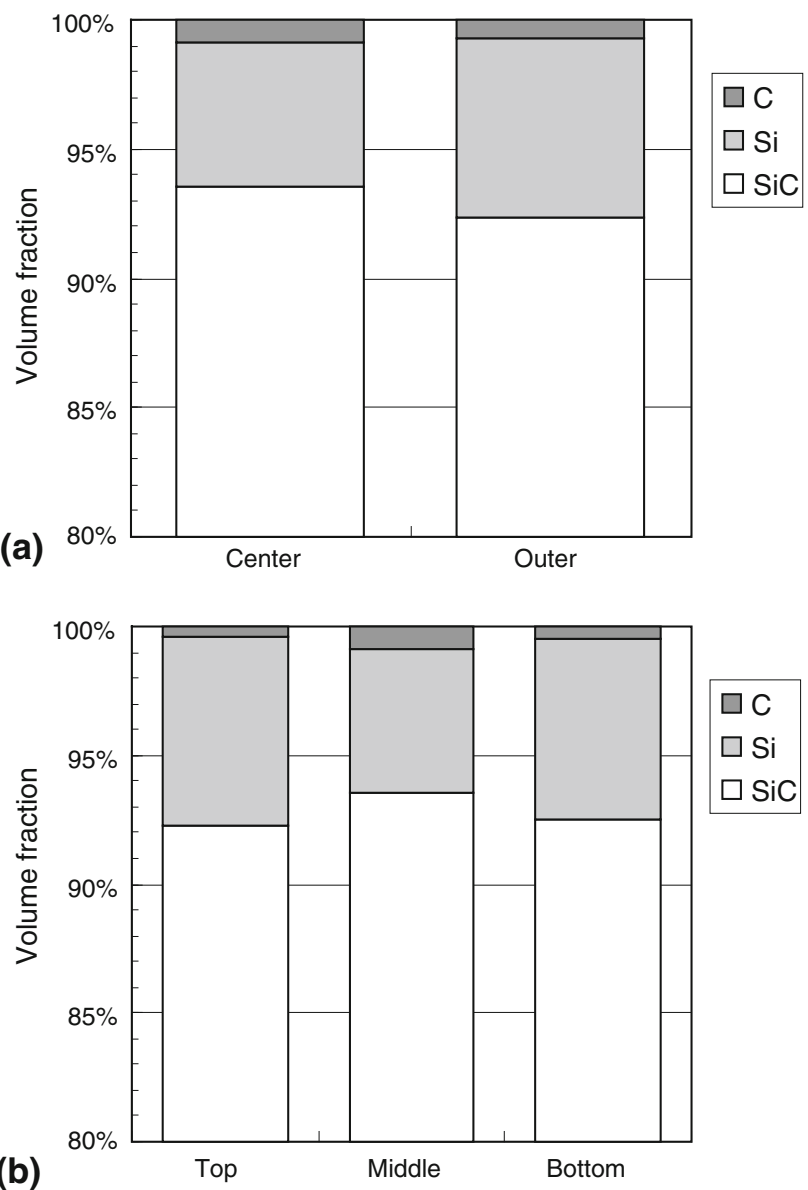

Fig. 11 Volume fractions of different phases under various sampling conditions obtained by image analysis

elastic modulus were higher in the in-plane direction than in the out-of-plane direction, it is presumed that the microstructure was more continuous in-plane than out-of-plane. The fact that the strength was higher in the outer positions than in the center suggests that the outer region may have had fewer defects, 


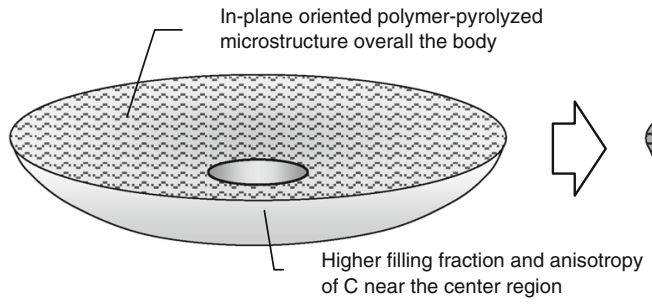

(a) carbon- based green body

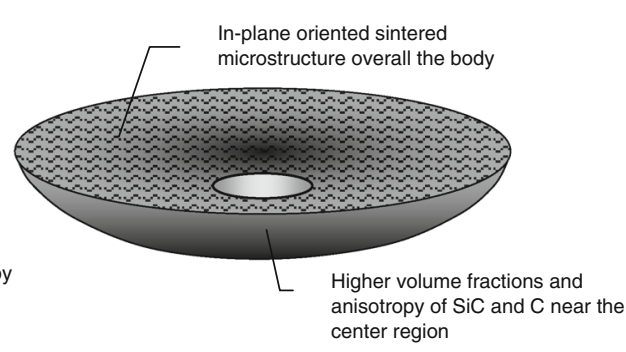

(b) Si impregnated and sintered body

Fig. 12 Schematic image of the microstructural tendency of the overall mirror body (a) before and (b) after reaction sintering

because of better conditions during the molten Si impregnation and/or gas purging. In addition, because the CTE was lower in the in-plane direction in the central region, it is inferred that microstructural conditions that lowered the CTE predominated under this condition.

Among the three phases that make up this material, the $\mathrm{SiC}$ phase had a higher strength and elastic modulus than the other phases, and the CTE was lower in the C phase than in the other phases. The high elastic modulus and low CTE in the middle of the material block are consistent with the results of the image analysis, which show a relatively high volume fraction of $\mathrm{SiC}$ and $\mathrm{C}$ phases in the middle. It is assumed that the filling fraction of carbon was relatively high in the middle in the green body stage, so it makes sense that the proportions of the $\mathrm{SiC}$ phase, which was produced from reaction sintering, and the unreacted $\mathrm{C}$ phase were high in the middle. A schematic image is shown in Fig. 12. Because the $\mathrm{SiC}$ phase is responsible for developing the elastic modulus and the $\mathrm{C}$ phase is responsible for reducing the CTE, the facts that the elastic modulus was higher in the in-plane direction than in the out-of-plane direction and that the CTE was lower in the in-plane direction than in the out-of-plane direction in the middle suggest that the material had higher continuity in the in-plane direction than in the out-of-plane direction. It is possible that some short carbon fibers in the green body and the pyrolytically produced carbon were oriented in the in-plane direction, which might have had some effect on the formation of the $\mathrm{SiC}$ phase after the reaction sintering. The anisotropy in the short fibers due to the effects of gravity or flows when the green body was formed is a possible explanation of why the anisotropy of the material was higher in the center region than in the outer region. Subsequently, various constituents in the outer region had high degrees of freedom to accommodate disturbances created by volume shrinkage or gas generation in the firing process, so the degree of orientation was lower in the outer positions. The fact that more pores were formed by gas generation from pyrolytically produced carbon during the reaction sintering in the middle, where carbon was denser, possibly explains why the strength was higher at the outer positions than at the center position. In addition, $\mathrm{Si}$ contributed to filling in the gaps in the $\mathrm{SiC}$ phase, which was responsible for the strength of the material.

Using the method described in this work, the overall characteristics of a large $\mathrm{SiC}$ mirror material block were identified based on an understanding of the correlation between the microstructure and its properties. This method will be useful for monitoring the defect distribution, uniformity, and reproducibility of a material block to identify any issues that may need to be improved during the development phase and for assessing the quality of flight products. However, it cannot be used to evaluate specific performance attributes such as the effect of thermal properties on the mirror accuracy. For such objectives, the related material properties in a mirror body would need to be investigated in much greater detail (Ref 14).

\section{Conclusions}

To develop a method for evaluating the material properties of $\mathrm{SiC}$ mirror bodies, we cut samples from the periphery of a prototype 800 -mm-diameter mirror body manufactured by reaction sintering. The major results obtained from the study are summarized as follows:

(i) By using this evaluation method, we were able to understand the overall variations in the microstructure and properties of the material block. We found that the volume fractions of the constituent phases differed between the middle and the other regions. In particular, the continuity of the microstructure was higher in the in-plane direction than in the out-of-plane direction, and the inplane and out-of-plane anisotropy in the middle was higher as compared to those in the other regions.

(ii) It would appear that the observed variations were closely associated with the orientation and filling fraction of the constituent material in the green body. To improve the homogeneity of the material block, it is important to secure an adequate level of homogeneity in the green body stage.

(iii) This evaluation method is thought to be effective as a means to understand the overall quality of the $\mathrm{SiC}$ material as a function of manufacturing conditions and to obtain feedback for improving those conditions. It would also be useful for assessing the quality of flight products.

\section{Acknowledgments}

We acknowledge Professor Hiroshi Hatta and Associate Senior Researcher Takuya Aoki of JAXA for providing helpful advice regarding sample evaluation at the beginning of the study. We also acknowledge JAXA staff members Ms. Rei Ueda and Mr. Kenichi Tomoda for supporting various experiments, including thermal expansion testing and flexural testing. We thank Mitsubishi Electric Corporation, for their assistance in sample preparation and providing information related to this matter, and Toray 
Research Center, Inc., for their assistance in material microstructure analysis. We take this opportunity to express our gratitude for their support and guidance.

\section{References}

1. R. Edeson, G.S. Aglietti, and A.R.L. Tatnall, Conventional Stable Structures for Space Optics: The State of the Art, Acta Astronaut., 2010, 66, p 13-32

2. J. Breysse, D. Castel, B. Laviron, D. Logut, and M. Bougoin, All-SiC Telescope Technology: Recent Progress and Achievements, Proceedings of the 5th International Conference on Space Optics (ICSO 2004), Mar 30, 2004 (France)

3. E. Sein, Y. Toulemont, J. Breysse, P. Deny, D. Chambure, T. Nakagawa, and M. Hirabayashi, A New Generation of Large SIC Telescopes for Space Applications, Proceedings of the SPIE 5528, Sep 30, 2004 (USA)

4. J.B. Hadaway, R. Eng, H.P. Stahl, J.R. Carpenter, J.R. Kegley, and W.D. Hogue, Cryogenic Performance of Lightweight $\mathrm{SiC}$ and $\mathrm{C} / \mathrm{SiC}$ Mirrors, Proceedings of the SPIE 5487, Oct 12, 2004 (Scotland)

5. Y. Yui, K. Goto, H. Kaneda, H. Katayama, M. Kotani, M. Miyamoto, M. Naitoh, T. Nakagawa, H. Saruwatari, M. Suganuma, H. Sugita, Y. Tange, S. Utsunomiya, Y. Yamamoto, and T. Yamawaki, Performance of Lightweight Large C/SiC Mirror, 7th International Conference on Space Optics (ICSO), Oct 14, 2008 (France)

6. M.R. Krödel, T. Ozaki, M. Kume, A. Furuya, Y.Y. Yui, H. Imai, H. Katayama, Y. Tange, T. Nakagawa, and H. Kaneda, Manufacturing and Performance Test of an 800-mm Space Optic, Proceedings of the SPIE 7018, Jul 14, 2008 (France)
7. H. Kaneda, M. Naitoh, T. Imai, Y. Tange, K. Enya, H. Katayama, K. Maruyama, T. Nakagawa, T. Onaka, M. Suganuma, R. Kano, Y. Kiriyama, T. Mori, and A. Takahashi, Cryogenic Optical Testing of an $800 \mathrm{~mm}$ Lightweight $\mathrm{C} / \mathrm{SiC}$ Composite Mirror Mounted on a $\mathrm{C} / \mathrm{SiC}$ Optical Bench, Appl. Opt., 2010, 49, p 3941-3948

8. H. Katayama, Y. Yamamoto, M. Miyamoto, H. Saruwatari, H. Kaneda, and Y. Tange, Measurement of Vibration Environment of $6 \mathrm{~m}$ Diameter Radiometer Thermal Vacuum Chamber in JAXA, Proceedings of the SPIE 7436, Aug 26, 2009 (USA)

9. M. Suganuma, H. Katayama, M. Naitoh, T. Imai, M. Miyamoto, H. Kaneda, Y. Tange, and T. Nakagawa, Development and Tests of Interferometry Facility in 6-m Diameter Radiometer Thermal Vacuum Chamber in Tsukuba Space Center, Proceedings of the SPIE 7731, Aug 10, 2010 (USA)

10. C. Devilliers and M.R. Krödel, CeSiC: A New Technology for Lightweight and Cost-Effective Space Instrument Structures and Mirrors, Proceedings of the SPIE 5494, Sep 24, 2004 (Scotland)

11. M.R. Krödel and T. Ozaki, HB-Cesic Composite for Space Optics and Structures, Proceedings of the SPIE 6666, Aug 26, 2007 (USA)

12. K. Enya, T. Nakagawa, H. Kaneda, T. Onaka, T. Ozaki, and M. Kume, Microscopic Surface Structure of C/SiC Composite Mirrors for Space Cryogenic Telescopes, Appl. Opt., 2007, 46, p 2049-2056

13. K. Enya, N. Yamada, T. Imai, Y. Tange, H. Kaneda, H. Katayama, M. Kotani, K. Maruyama, M. Naitoh, T. Nakagawa, T. Onaka, M. Suganuma, T. Ozaki, M. Kume, and M.R. Krödel, High-Precision CTE Measurement of Hybrid $\mathrm{C} / \mathrm{SiC}$ Composite for Cryogenic Space Telescopes, Cryogenics, 2012, 52, p 86-89

14. M. Kotani, T. Imai, H. Katayama, Y. Yui, Y. Tange, H. Kaneda, T. Nakagawa, and K. Enya, Quality Evaluation of Spaceborne SiC Mirrors (I): Analytical Examination of the Effects on Mirror Accuracy by Variation in the Thermal Expansion Property of the Mirror Surface, Appl. Opt., 2013, 52, p 4797-4805 